\title{
Bafilomycin A1 inhibits the growth and metastatic potential of the BEL-7402 liver cancer and HO-8910 ovarian cancer cell lines and induces alterations in their microRNA expression
}

\author{
XIAODONG LU ${ }^{1,2}$, LUFANG CHEN ${ }^{1}$, YUANYUAN CHEN ${ }^{1}$, QIXIANG SHAO ${ }^{1}$ and WENXIN QIN ${ }^{2}$ \\ ${ }^{1}$ School of Medicine, Jiangsu University, Zhenjiang, Jiangsu 212013; ${ }^{2}$ State Key Laboratory of Oncogenes and Related Genes, \\ Shanghai Cancer Institute, Shanghai 200032, P.R. China
}

Received October 9, 2014; Accepted September 1, 2015

DOI: $10.3892 / \mathrm{etm} .2015 .2758$

\begin{abstract}
The vacuolar $\mathrm{H}^{+}$-ATPase (V-ATPase) is commonly highly activated in cancer cells and is a potential target of anti-cancer therapy. Bafilomycin A1 is a specific inhibitor of the c subunit of V-ATPase. In the present study, the effects of bafilomycin A1 on the BEL-7402 hepatocellular carcinoma and HO-8910 ovarian cancer cell lines were respectively studied. In addition, the bafilomycin A1-induced alterations in the mRNAs and microRNAs (miRNAs) in the cells were detected using microarray methods. The results demonstrated that the growth of the two cell lines was retarded and the metastatic potential was inhibited by bafilomycin A1. Transmission electron microscopy and assays of capsase- 3 and -9 suggested that bafilomycin A1 induced apoptosis. Gene Ontology analysis of the microarrays of mRNA-miRNA integrity showed altered pathways following bafilomycin A1 treatment, including pathways regulating glucose or lipid metabolism, DNA repair or duplication and lysosomes. Quantitative polymerase chain reaction analysis confirmed that miR-923, miR-1246, miR-149*, miR-638 and miR-210 were upregulated and miR-99a, miR-181a-2* and miR-339-5p were downregulated following bafilomycin A1 treatment. The overlapped altered miRs may be effective targets for the two types of solid tumor, and may have potential for application to the treatment of other types of solid tumor.
\end{abstract}

Correspondence to: Professor Xiaodong Lu or Professor Qixiang Shao, School of Medicine, Jiangsu University, 301 Xue-fu Road, Zhenjiang, Jiangsu 212013, P.R. China

E-mail: lu_xdg@hotmail.com

E-mail: shao_qx@ujs.edu.cn

Key words: bafilomycin A1, vacuolar $\mathrm{H}^{+}$-ATPase, BEL-7402, HO-8910, apoptosis, microRNA

\section{Introduction}

The vacuolar $\mathrm{H}^{+}$-ATPase (V-ATPase) is a transmembrane enzyme that actively pumps protons to the extracellular matrix or intracellular compartments (e.g. endosomes, lysosomes and secretory vesicles), in order to regulate the alkalinization of the cytosol and acidification of cellular compartments (1-3). The V-ATPase is widely distributed in eukaryotic cells and exhibits particularly high levels of activity in cancer cells (4). The significance of the excessive activity of the V-ATPase in cancer cells is to maintain the slightly alkaline intracellular $\mathrm{pH}$, in order to promote the survival of tumor cells when excess acidosis is produced due to the 'Warburg effect'; furthermore, the acidified extracellular environment facilitates metastasis $(5,6)$. High levels of V-ATPase activity therefore promote the malignancy of tumors. Functions of the V-ATPase have been discovered over many years and include regulating signal transduction (7), glucose metabolism (8), lysosome functions (9), endosomal trafficking $(10,11)$ and the endoplasmic reticulum stress response (12). Considerable evidence supports the suggestion that the V-ATPase represents a potential target of anti-tumor therapy (13-16). Bafilomycin A1 is a specific inhibitor of the c subunit of the V-ATPase and has been found to inhibit the proliferation and metastasis of cancer cells (17).

MicroRNAs (miRNAs), the intrinsic, small, non-protein-coding RNAs that effectively regulate gene expression, play important roles in determining the proliferation or apoptosis of cancer cells $(18,19)$. The aim of the present study was to investigate the effects of bafilomycin A1 on the BEL-7402 hepatocellular carcinoma and HO-8910 ovarian cancer cell lines and to use microarray and reverse transcription-quantitative polymerase chain reaction (RT-qPCR) techniques to explore the altered pathways and miRNA expression induced by bafilomycin A1 in these cell lines.

\section{Materials and methods}

Cells and chemicals. The human BEL-7402 hepatocellular carcinoma and HO-8910 ovarian cancer cell lines were purchased from the Cell Bank of the Chinese Academy of Science (Shanghai, China) and maintained in RPMI-1640 medium (Gibco-BRL, Rockville,MD,USA) supplemented with 
$10 \%$ fetal calf serum (FCS; Hangzhou Sijiqing Bioengineering Material Co., Ltd., Hangzhou, China), $100 \mathrm{U} / \mathrm{ml}$ penicillin and $100 \mu \mathrm{g} / \mathrm{ml}$ streptomycin. Cells were incubated at $37^{\circ} \mathrm{C}$ in a $5 \% \mathrm{CO}_{2}$ and $95 \%$ air atmosphere and were subcultured at $\sim 80 \%$ confluence. Bafilomycin A1 was purchased from Sigma-Aldrich (St. Louis, MO, USA).

Water-soluble tetrazolium salt (WST)-1 cell proliferation assay. Cell proliferation and viability were analyzed using the WST-1 Cell Proliferation Assay kit (Beyotime Institute of Biotechnology, Haimen, China) according to the manufacturer's instructions. Briefly, cells were harvested using $0.05 \%$ trypsin and suspended in culture medium containing $10 \%$ FCS at a concentration of $5 \times 10^{4} \mathrm{cells} / \mathrm{ml}$, and $200 \mu \mathrm{l}$ suspension was added to each well of a 96-well plate. Cells were cultured for $20 \mathrm{~h}$ for adhesion. Bafilomycin A1 was added to the wells at the final concentrations of 200, 400 and $800 \mathrm{nM}$, in triplicate. At 24,48 and $72 \mathrm{~h}, 20 \mu \mathrm{l}$ WST-1 was added to the cells. Following incubation at $37^{\circ} \mathrm{C}$ for $4 \mathrm{~h}$, the plates were read to determine the optical density (OD) at $435 \mathrm{~nm}$ with $675 \mathrm{~nm}$ reference using a spectrophotometer.

Soft-agar colony formation assay. Soft agar assays were performed in a 6-well plate. Each well contained $2 \mathrm{ml} \mathrm{0.5 \%}$ agarose (Sigma-Aldrich) at the bottom and $2 \mathrm{ml} 0.35 \%$ agarose containing $1 \times 10^{4}$ cells on top. The medium used was the aforementioned culture media with serial concentrations of bafilomycin A1. Each concentration was set in triplicate. Cells were grown at $37^{\circ} \mathrm{C}$ in a $5 \% \mathrm{CO}_{2}$ and $95 \%$ air atmosphere for 14 days. Colonies were counted under a phase-contrast microscope (XD-202; Nanjing Jiangnan Novel Optics, Co., Ltd., Nanjing, China). The relative proliferation ratio was calculated using the following formula: Relative proliferation ratio $=$ Number of treated/Number of control.

Capsase-3 and -9 assays. A total of $1 \times 10^{6}$ BEL-7402 and HO-8910 cells, respectively, were seeded in a 6-well plate (Corning, Inc., Corning, NY, USA) and cultured with the serial concentrations of bafilomycin A1 in RPMI-1640 medium with $10 \% \mathrm{FCS}$ at $37^{\circ} \mathrm{C}$ in a $5 \% \mathrm{CO}_{2}$ and $95 \%$ air atmosphere for 24,48 and $72 \mathrm{~h}$. The activities of caspase-3 and -9 in the cellular extracts were determined by colorimetric assay, using commercial Caspase-3 and -9 Activity kits (Beyotime Institute of Biotechnology) according to the manufacturer's instructions. The protein concentrations of the samples were determined using a Bicinchoninic Acid Assay kit (Beyotime Institute of Biotechnology). The relative enzyme activity was calculated using the following formula: Relative enzyme activity $=$ enzyme activity of treated cells per $\mu \mathrm{g}$ protein/enzyme activity of control cells per $\mu \mathrm{g}$ protein.

Transmission electron microscopy (TEM). A total of 1x10 ${ }^{6}$ BEL-7402 and HO-8910 cells, respectively, were seeded and cultured with the serial concentrations of bafilomycin $\mathrm{A} 1$ in RPMI-1640 medium with $10 \% \mathrm{FCS}$ at $37^{\circ} \mathrm{C}$ in a $5 \% \mathrm{CO}_{2}$ and $95 \%$ air atmosphere for $48 \mathrm{~h}$. The cells were washed with phosphate-buffered saline (PBS), harvested using $0.03 \%$ trypsin, re-washed with PBS to remove the trypsin and fixed in $2.5 \%$ glutaraldehyde at $4{ }^{\circ} \mathrm{C}$ overnight. The cells were then centrifuged at $500 \mathrm{xg}$ for $5 \mathrm{~min}$ to remove the glutaraldehyde and postfixed in $1 \% \mathrm{OsO}_{4}$ for $2 \mathrm{~h}$. Following postfixation, the cells were washed with PBS, dehydrated in ethanol and embedded in Epon 812 (Sigma-Aldrich). Ultrathin sections were sliced using a Leica-Reichert Ultracut ultramicrotome (Leica Microsystems GmbH, Wetzlar, Germany), stained with $2 \%$ aqueous uranyl acetate (Sigma-Aldrich) and lead citrate (Sigma-Aldrich) and observed with an HT-7700 electron microscope (Hitachi, Ltd., Tokyo, Japan).

Cell invasion assay. The cells were cultured in the aforementioned complete culture medium until reaching $80 \%$ confluence and then starved in serum-free medium for $8 \mathrm{~h}$. The cells were subsequently washed with PBS, harvested, diluted to the concentration of $1 \times 10^{5}$ cells $/ \mathrm{ml}$ in $0.1 \%$ serum RPMI-1640 medium with serial concentrations of bafilomycin A1 and then plated in an invasion chamber $\left(1 \times 10^{4}\right.$ cells in $100 \mu$ l medium per well) of a Cell Invasion Assay kit (Chemicon International, Inc., Temecula, CA, USA). The invasion chamber was inserted into the feeder tray, which contained $150 \mu 1 \quad 10 \%$ serum RPMI-1640 medium per well. The plate was incubated for $20 \mathrm{~h}$ at $37^{\circ} \mathrm{C}$ in $5 \% \mathrm{CO}_{2}$ and $95 \%$ air. The cells that had invaded through the membrane were washed with detachment solution and stained with lysis buffer/dye solution, and the fluorescence absorbance was measured in a luminescence spectrometer at $480 / 520 \mathrm{~nm}$.

Total RNA extraction. Total RNA of the control cells and cells that had been exposed to $400 \mathrm{nM}$ bafilomycin A1 for $48 \mathrm{~h}$ was extracted using TRIzol ${ }^{\circledR}$ reagent (Invitrogen, Life Technologies, Carlsbad, CA, USA) in accordance with the manufacturer's instructions. RNA concentration and purity were determined via ultraviolet spectrometry, measuring the OD at 260 and $280 \mathrm{~nm}$.

Microarray of mRNA and miRNA. Total extracted RNA was subjected to mRNA and miRNA microarray using the Affymetrix GeneChip ${ }^{\circledR}$ Human Gene and miRNA Arrays (Affymetrix, Inc., Santa Clara, CA, USA), respectively, at Genminix Informatics Ltd., Co. (Shanghai, China) according to the instructions provided with the Affymetrix arrays. Briefly, RNA was extracted from cells following treatment with $400 \mathrm{nM}$ bafilomycin A1 for $48 \mathrm{~h}$ using TRIzol reagent (Invitrogen, Life Technologies). The quantity and purity of the RNA were assessed using a NanoDrop ND-1000 spectrophotometer (NanoDrop Technologies, Inc., Wilmington, DE, USA). The purity and integrity of each extracted RNA met the following requirements: A260/A280 >1.6, A260/A230 >1 and RNA Integrity Number $>5$. The small RNA fraction indicated an abundance of RNA $<200 \mathrm{nt}$, compared with total RNA using Agilent RNA 6000 Nano assay (Agilent Technologies, Inc., Santa Clara, CA, USA), and was acceptable for miRNA assay. The possibility of genomic DNA contamination was excluded using gel electrophoresis. A total of $2 \mu \mathrm{g}$ RNA from each group was respectively converted into cyanine-5-labeled target cRNA, hybridized to the Affymetrix GeneChip Human Gene and miRNA Arrays, respectively, using the Affymetrix GeneChip Fluidics Station 450 (Affymetrix, Inc.), and scanned with an Affymetrix GeneChip Scanner 3000 7G (Affymetrix, Inc.). Following normalization, differentially expressed mRNAs or miRNAs were established at $\log 2$ (fold change) $>1$ and $\mathrm{P}<0.05$. 
A
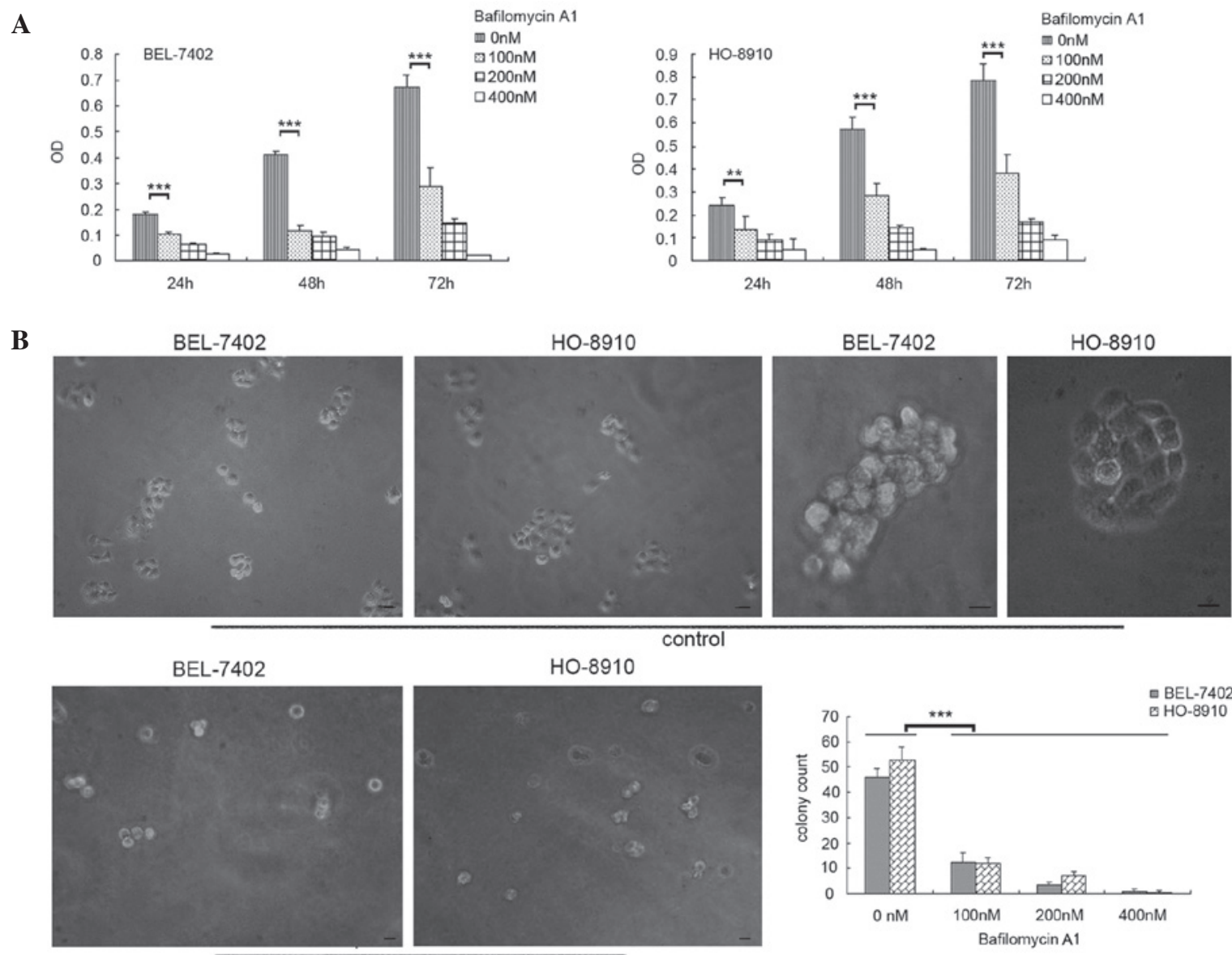

100nM Bafilomycin A1 treated

Figure 1. Bafilomycin A1 inhibits BEL-7402 and HO-8910 cell proliferation. (A) Water-soluble tetrazolium salt-1 assay showed that the proliferation of the two cell lines was retarded. (B) Soft-agar colony formation assay showed that the total numbers of colonies of treated cells were significantly less than those of the control (bottom right). Few micro- or middle-colonies (containing 3-5 or 6-10 cells/colony, respectively) and non macro-colonies ( $>10$ cells) could be observed among the treated cells, contrasting with the control. Scale bar, $10 \mu \mathrm{m} ;{ }^{* *} \mathrm{P}<0.01$ and ${ }^{* * *} \mathrm{P}<0.001$. OD, optical density.

RT-qPCR analysis of miRNA. RNA was transcribed into cDNA using the miScript II RT kit (Qiagen, Hilden, Germany). The reaction components were as follows: Total RNA, $1 \mu \mathrm{g}$; miScript HiSpec Buffer, $4 \mu \mathrm{l}$; Nucleic Acid Mix, $2 \mu 1$; miScript Reverse Transcriptase Mix, $2 \mu \mathrm{l}$; RNase-free $\mathrm{H}_{2} \mathrm{O},<20 \mu \mathrm{l}$. The reaction was performed at $37^{\circ} \mathrm{C}$ for $60 \mathrm{~min}$ and $95^{\circ} \mathrm{C}$ for $5 \mathrm{~min}$ on an ABI PCR 9700 system (Applied Biosystems, Foster City, CA, USA). cDNA was diluted in $80 \mu \mathrm{l}$ nuclease-free $\mathrm{H}_{2} \mathrm{O}$ for the addition of LightCycler ${ }^{\circledR} 480$ SYBR Green I Master (Roche Diagnostics, Indianapolis, IN, USA). The reaction system for the qPCR was as follows: LightCycler 480 SYBR Green I Master, $5 \mu \mathrm{l}$; forward primer, $0.2 \mu \mathrm{l}$; reverse primer, $0.2 \mu \mathrm{l}$ (provided by RiboBio Co., Ltd., Guangzhou, China); cDNA, $1 \mu \mathrm{l}$; nuclease-free $\mathrm{H}_{2} \mathrm{O}, 3.6 \mu \mathrm{l}$. The PCR was run using an ABI 7500 Fast system (Applied Biosystems), as follows: $94^{\circ} \mathrm{C}$ for $2 \mathrm{~min}$, followed by 40 cycles of $94^{\circ} \mathrm{C}$ for $15 \mathrm{sec}, 62^{\circ} \mathrm{C}$ for $40 \mathrm{sec}$ and $70^{\circ} \mathrm{C}$ for $40 \mathrm{sec}$. The mRNA expression was normalized against the expression of U6. All samples were analyzed in triplicate. Relative expression was calculated using the comparative threshold cycle $(\mathrm{Ct})$ method and was indicated as the fold change, as follows: Fold change $=2^{-(\Delta \text { Cttreated }-\Delta \text { Ctcontrol })}$.

Data analysis. Microarray data were analyzed by Genminix Informatics Ltd., Co. The integrated analysis of the differ- ential mRNA and miRNA expression due to treatment with bafilomycin A1 was performed to reveal the target genes of the drug via miRNA regulation. The target genes of the differential miRNA were predicted using bioinformatics [TargetScan (http://www.targetscan.org/) and MicroCosm Targets (http://www.ebi.ac.uk/enright-srv/microcosm/htdocs/targets)]. The overlapping altered genes in the mRNA microarray and the miRNA predicted genes were subjected to Gene Ontology (GO) term and pathway analyses, in order to organize the genes into hierarchical categories and uncover the altered pathways and miRNA-gene regulatory network upon which bafilomycin A1 likely has an effect.

Statistical analysis. Statistical analysis was carried out using Microsoft Excel software (Microsoft Corp., Redmond, WA, USA). Data were compared using a two-tailed Student's $t$-test, and $\mathrm{P}<0.05$ was considered to indicate a statistically significant difference.

\section{Results}

Bafilomycin Al inhibits BEL-7402 and HO-8910 cell proliferation. WST-1 cell proliferation and soft-agar colony formation assays were performed to determine the effect of 
Table I. Numbers of altered genes and miRNAs.

\begin{tabular}{lccccc}
\hline & \multicolumn{2}{c}{ Number of altered genes } & & \multicolumn{2}{c}{ Number of altered miRNAs } \\
\cline { 2 - 3 } \cline { 5 - 6 } Cell line & Upregulated & Downregulated & & Upregulated & Downregulated \\
\hline BEL-7402 & 252 & 127 & & 12 & 8 \\
HO-8910 & 126 & 57 & 18 & 20 \\
\hline
\end{tabular}

miRNA, microRNA.
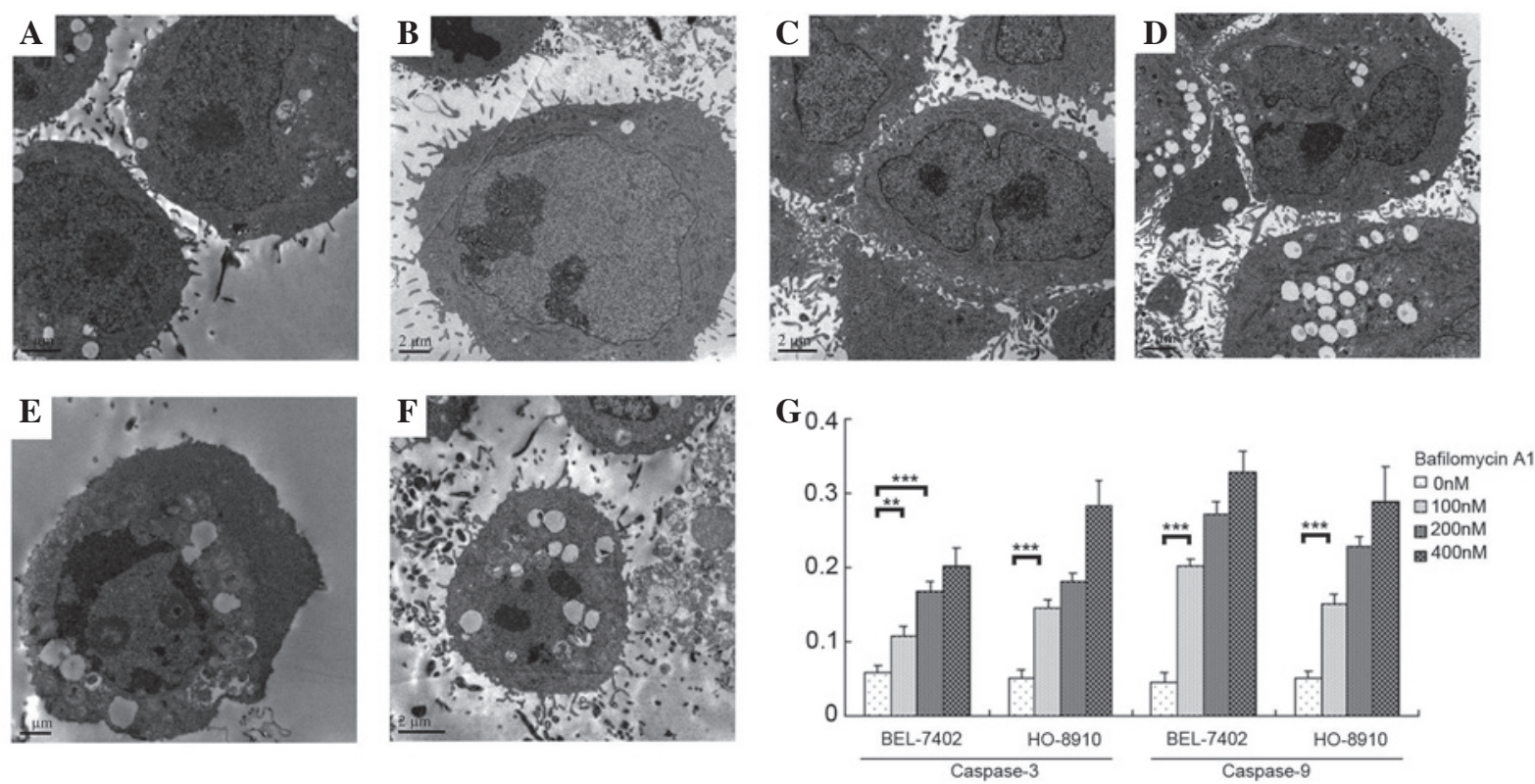

Figure 2. Transmission electron microscopy observations of bafilomycin A1-treated BEL-7402 and HO-8910 cells at 48 h. (A and B) Non bafilomycin A1-treated BEL-7402 and HO-8910 cells, respectively. (C and D) BEL-7402 and HO-8910 cells, respectively, treated with $100 \mathrm{nM}$ bafilomycin A1 exhibited indentations and folding of the nuclear membrane. (E) BEL-7402 cells treated with $200 \mathrm{nM}$ bafilomycin A1 showed chromosome condensation at the periphery of the nuclear membrane. (F) HO-8910 cells treated with $200 \mathrm{nM}$ bafilomycin A1 exhibited a condensed nucleolus and highly vacuolar cytoplasm. (G) Assays for capsase-3 and -9, two important enzymes involved in apoptosis, showed significant increases following bafilomycin A1-treatment. ${ }^{* *} \mathrm{P}<0.01$ and ${ }^{* * * *} \mathrm{P}<0.001$.

bafilomycin A1 on BEL-7402 and HO-8910 cells. The results indicated that the proliferation of the two cell lines was inhibited by bafilomycin A1, as shown in Fig. 1A. The inhibitory effect was also evident in the soft-agar colony formation assay (Fig. 1B). Compared with the control cells, few micro- or middle-colonies (containing 3-5 or 6-10 cells/colony, respectively) and non-macro-colonies (containing $>10$ cells) could be observed among the bafilomycin A1-treated cells, and the total numbers of colonies were significantly reduced.

TEM observations of bafilomycin Al-treated BEL-7402 and HO-8910 cells. TEM was used to capture images of the cells treated with various concentrations of bafilomycin $\mathrm{A} 1$ at 48 h. Non-bafilomycin A1-treated BEL-7402 and HO-8910 cells are shown in Fig. 2A and B, respectively. BEL-7402 and HO-8910 cells treated with $100 \mathrm{nM}$ bafilomycin A1 (Fig. 2C and $\mathrm{D}$, respectively) exhibited indentations and folding of the nuclear membrane, whereas BEL-7402 and HO-8910 cells treated with $200 \mathrm{nM}$ bafilomycin A1 (Fig. 2E and F, respectively) exhibited chromosome condensation at the periphery of the nuclear membrane, condensed nuclei and

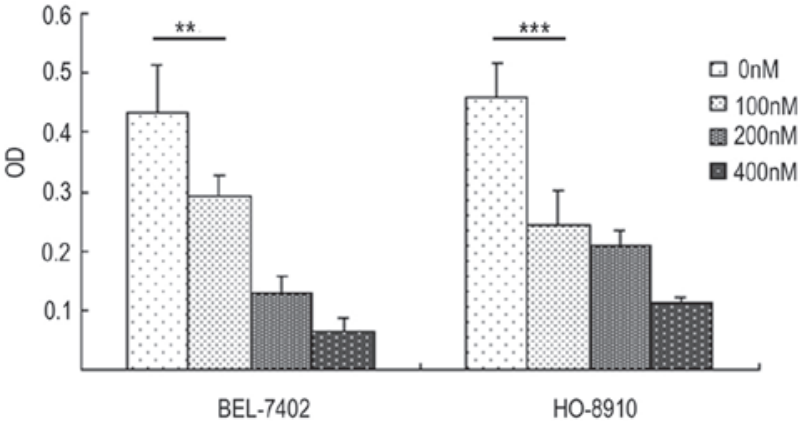

Figure 3. Bafilomycin A1 suppresses the invasion of BEL-7402 and HO-8910 cells. Cell invasion assay showed that the invasive potential of BEL-7402 and HO-8910 cells was significantly suppressed following bafilomycin A1 treatment. ${ }^{* *} \mathrm{P}<0.01$ and ${ }^{* * * *} \mathrm{P}<0.001$. OD, optical density.

vacuolar cytoplasms. The images suggested an apoptotic response to the cellular toxicity. Furthermore, the assays for capsase- 3 and -9 , which are two important enzymes involved in apoptosis, showed significant increases following bafilomycin A1-treatment (Fig. 2G). 

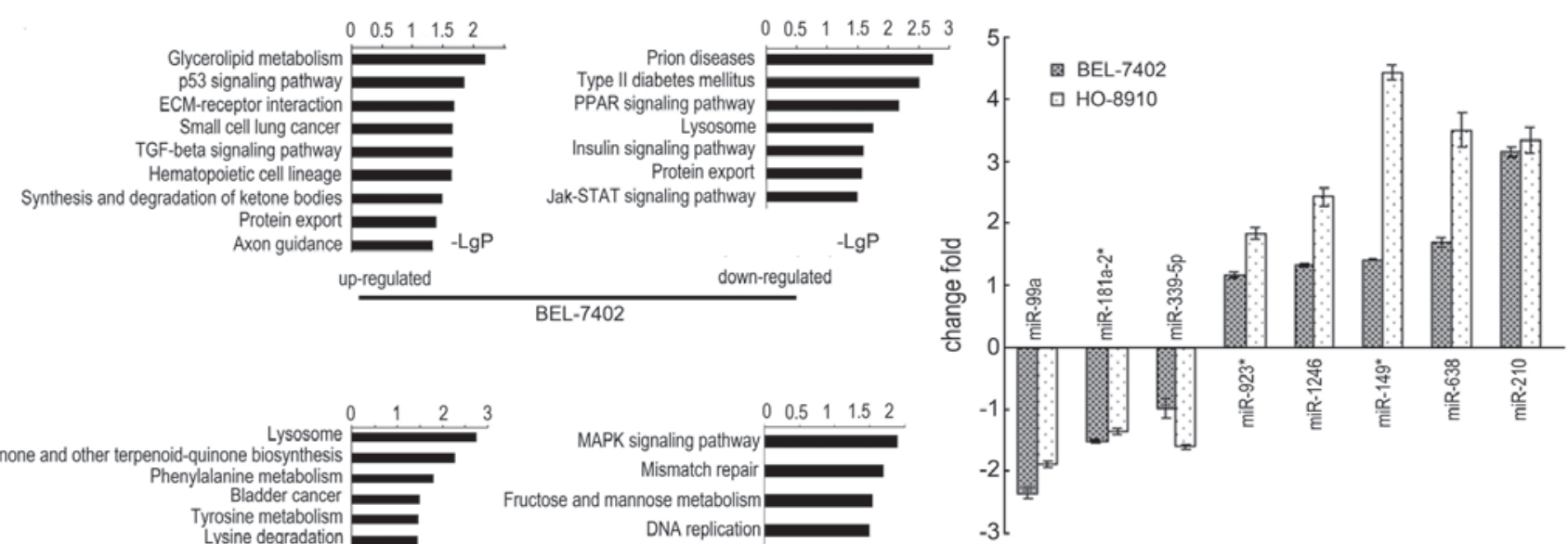

Figure 4. Bafilomycin A1-induced altered pathways and miRNAs. (A) miRNA-mRNA integrity analysis demonstrated the significantly altered pathways in the BEL-7402 and HO-8910 cell lines. (B) Quantitative polymerase chain reaction assay confirmed the eight common significantly altered miRNAs following treatment with bafilomycin A1, $\log 2$ fold change is shown (each of the eight, $\mathrm{P}<0.001$ vs. the control). miRNA, microRNA; ECM, extracellular matrix; TFG, transforming growth factor; PPAR, peroxisome proliferator-activated receptor; Jak, Janus kinase; STAT, signal transducer and activator of transcription; mTOR, mammalian target of rapamycin; MAPK, mitogen-activated protein kinase.

Bafilomycin Al suppresses the invasion of BEL-7402 and $\mathrm{HO}-8910$ cells. According to the cell invasion assays, the invasive potential of the BEL-7402 and HO-8910 cells was significantly suppressed with the bafilomycin A treatment, as shown in Fig. 3.

Bafilomycin Al induces alterations in certain pathways. The numbers of altered mRNAs and miRNAs in BEL-7402 and HO-8910 cells $48 \mathrm{~h}$ after exposure to $400 \mathrm{nM}$ bafilomycin A1 are shown in Table I. The overlapping altered genes in the mRNA microarray and the miRNA predicted genes were determined, and GO analysis was performed. The altered pathways are shown in Fig. 4A. After treatment with $400 \mathrm{nM}$ bafilomycin A1 for $48 \mathrm{~h}$, the following miRNAs were altered in the two cell lines: miR-923, miR-1246, miR-149*, miR-638 and miR-210 were significantly upregulated, whereas miR-99a, miR-181a-2* and miR-339-5p were significantly downregulated. These results were confirmed by qPCR (Fig. 4B).

\section{Discussion}

The V-ATPase is typically highly activated in cancer cells, in which it has the following functions: Regulating cell proliferation, apoptosis and autophagy; facilitating cancer metastasis; contributing to the acquirement of drug resistance; and affecting signal transduction (20-23). Inhibitors of the V-ATPase, therefore, are promising anti-cancer chemicals. In the present study, the inhibitory effects of bafilomycin A1 on two cell lines, BEL-7402 and HO-8910, were investigated, and it was found that bafilomycin A1 suppressed the proliferation, induced the apoptosis and attenuated the invasive potential of the cells.

Following exposure to $400 \mathrm{nM}$ bafilomycin A1 for $48 \mathrm{~h}$, an mRNA-miRNA microarray integrity analysis was performed to reveal the altered pathways, among which several were associated with glucose or lipid metabolism, including glycerolipid metabolism, insulin signaling, fructose and mannose metabolism, phenylalanine metabolism and the synthesis and degradation of ketone bodies (Fig. 4); this finding may result from the coupling of glucose metabolism and regulation of the cytosolic/extracellular $\mathrm{pH}$ gradient by the V-ATPase. By dysregulating the V-ATPase, bafilomycin A1 thus affected the metabolism of glucose $(8,24)$. Pathways associated with DNA repair or duplication were also found to be altered, including the p53 signaling pathway, nucleotide excision repair, and DNA replication and mismatch repair, which suggested the toxicity of bafilomycin A1 and was consistent with the retarded proliferation. A number of altered signal pathways induced by bafilomycin A1, which were suggested to be highly related to the functions of V-ATPase, have been previously reported, such as mammalian target of rapamycin (25), AMP-activated protein kinase (26) and transforming growth factor- $\beta$ (27). The present study is the first to additionally report Janus kinase-signal transducer and activator of transcription and peroxisome proliferator-activated receptor (Fig. 4).

Notably, the lysosome pathway was altered in both cell lines, but in a contrasting manner, i.e., in BEL-7402 cells the pathway was downregulated, whereas in HO-8910 cells the pathway was upregulated. Previous studies have suggested that the functions of lysosomes and the V-ATPase are closely associated and that their interactions are involved in the regulations of autophagy and apoptosis $(9,28)$.

Liver cancer and ovarian cancer are different types of solid cancer; men are more susceptible to the former, while the latter is female specific. This is one of the reasons that these two cell lines were selected for the present study; in order to expand their contrasts. However, bafilomycin A1 displayed a high efficiency of inhibitory effects on both tumor types in vitro. Although the inhibitory effects of bafilomycin A1 in the two cancer cell lines were similar, the cellular pathways 
involved in the action of bafilomycin A1 were not identical, as an miRNA microarray showed that 8 miRNAs were altered in both cell lines. miRNAs serve an advanced regulatory function in cellular pathways, with one miRNA typically impacting numerous mRNAs. The miRNAs that are commonly altered by bafilomycin A1 in the two cell lines may represent promising targets for anti-cancer therapies. However, further studies are required to determine whether the miRNAs exhibit similar effects in other types of solid tumor.

\section{Acknowledgements}

This study was supported by grants from the State Key Laboratory of Oncogenes and Related Genes (no. 90-10-02), the Clinical Medicine Science and Technology Project of Jiangsu Province China (no. BL2013024) and the National Nature Science Foundation of China (no. 81372404).

\section{References}

1. Nishi T and Forgac M: The vacuolar $\left(\mathrm{H}^{+}\right)$-ATPases-nature's most versatile proton pumps. Nat Rev Mol Cell Biol 3: 94-103, 2002.

2. Marshansky V, Rubinstein JL and Grüber G: Eukaryotic V-ATPase: Novel structural findings and functional insights. Biochimica Biophy Acta 1837: 857-879, 2014.

3. Breton S and Brown D: Regulation of luminal acidification by the V-ATPase. Physiology (Bethesda) 28: 318-329, 2013.

4. Sun-Wada GH and Wada Y: Vacuolar-type proton pump ATPases: Acidification and pathological relationships. Histol Histopathol 28: 805-815, 2013.

5. Barar J and Omidi Y: Dysregulated $\mathrm{pH}$ in tumor microenvironment checkmates cancer therapy. BioImpacts 3: 149-162, 2013

6. Chung C, Mader CC, Schmitz JC, Atladottir J, Fitchev P, Cornwell ML, Koleske AJ, Crawford SE and Gorelick F: The vacuolar-ATPase modulates matrix metalloproteinase isoforms in human pancreatic cancer. Lab Invest 91: 732-743, 2011.

7. Sennoune SR and Martinez-Zaguilan R: Vacuolar H(+)-ATPase signaling pathway in cancer. Curr Protein Pept Sci 13: 152-163, 2012.

8. Fogarty FM, O'Keeffe J, Zhadanov A, Papkovsky D, Ayllon V and O'Connor R: HRG-1 enhances cancer cell invasive potential and couples glucose metabolism to cytosolic/extracellular $\mathrm{pH}$ gradient regulation by the vacuolar-H(+) ATPase. Oncogene 33: 4653-4663, 2014.

9. Nakashima S, Hiraku Y, Tada-Oikawa S, Hishita T, Gabazza EC, Tamaki S, Imoto I, Adachi Y and Kawanishi S: Vacuolar $\mathrm{H}^{+}$-ATPase inhibitor induces apoptosis via lysosomal dysfunction in the human gastric cancer cell line MKN-1. J Biochem 134: 359-364, 2003

10. Yan Y, Denef $N$ and Schüpbach T: The vacuolar proton pump, $\mathrm{V}$-ATPase, is required for notch signaling and endosomal trafficking in drosophila. Dev Cell 17: 387-402, 2009.

11. Marshansky V and Futai M: The V-type $\mathrm{H}^{+}$-ATPase in vesicular trafficking: Targeting, regulation and function. Curr Opin Cell Biol 20: 415-426, 2008.

12. Lee GH, Kim DS, Kim HT, Lee JW, Chung CH, Ahn T, Lim JM, Kim IK, Chae HJ and Kim HR: Enhanced lysosomal activity is involved in Bax inhibitor-1-induced regulation of the endoplasmic reticulum (ER) stress response and cell death against ER stress: Involvement of vacuolar $\mathrm{H}^{+}$-ATPase (V-ATPase). J Biol Chem 286: 24743-24753, 2011.
13. Avnet S, Di Pompo G,Lemma S, Salerno M,Perut F, Bonuccelli G, Granchi D, Zini N and Baldini N: V-ATPase is a candidate therapeutic target for Ewing sarcoma. Biochim Biophys Acta 1832: 1105-1116, 2013

14. Hernandez A, Serrano-Bueno G, Perez-Castineira JR and Serrano A: Intracellular proton pumps as targets in chemotherapy: V-ATPases and cancer. Curr Pharm Des 18: 1383-1394, 2012.

15. Graham RM, Thompson JW and Webster KA: Inhibition of the vacuolar ATPase induces Bnip3-dependent death of cancer cells and a reduction in tumor burden and metastasis. Oncotarget 5: 1162-1173, 2014

16. Luciani F, Spada M, De Milito A, Molinari A, Rivoltini L, Montinaro A, Marra M, Lugini L, Logozzi M, Lozupone F, et al: Effect of proton pump inhibitor pretreatment on resistance of solid tumors to cytotoxic drugs. J Nat Cancer Inst 96: 1702-1713, 2004.

17. Morimura T, Fujita K, Akita M, Nagashima M and Satomi A: The proton pump inhibitor inhibits cell growth and induces apoptosis in human hepatoblastoma. Pediat Surg Int 24: 1087-1094, 2008.

18. Baer C, Claus R and Plass C: Genome-wide epigenetic regulation of miRNAs in cancer. Cancer Res 73: 473-477, 2013.

19. Wang Y and Taniguchi T: MicroRNAs and DNA damage response: Implications for cancer therapy. Cell Cycle 12: 32-42, 2013.

20. von Schwarzenberg K, Wiedmann RM, Oak P, Schulz S, Zischka H, Wanner G, Efferth T, Trauner D and Vollmar AM: Mode of cell death induction by pharmacological vacuolar $\mathrm{H}^{+}$-ATPase (V-ATPase) inhibition. J Biol Chem 288: 1385-1396, 2013.

21. Ohta T, Arakawa H, Futagami F, Fushida S, Kitagawa H, Kayahara M, Nagakawa T, Miwa K, Kurashima K, Numata M, et al: Bafilomycin A1 induces apoptosis in the human pancreatic cancer cell line Capan-1. J Pathol 185: 324-330, 1998

22. Hendrix A, Sormunen R, Westbroek W, Lambein K, Denys H, Sys G, Braems G, Van den Broecke R, Cocquyt V, Gespach C, et al: Vacuolar $\mathrm{H}^{+}$ATPase expression and activity is required for Rab27B-dependent invasive growth and metastasis of breast cancer. Int J Cancer 133: 843-854, 2013.

23. Lu Q, Lu S, Huang L, Wang T, Wan Y, Zhou CX, Zhang C, Zhang $\mathrm{Z}$ and Li X: The expression of V-ATPase is associated with drug resistance and pathology of non-small-cell lung cancer. Diagn Pathol 8: 145, 2013.

24. Dechant R, Binda M, Lee SS, Pelet S, Winderickx J and Peter M: Cytosolic $\mathrm{pH}$ is a second messenger for glucose and regulates the PKA pathway through V-ATPase. EMBO J 29: 2515-2526, 2010.

25. Valapala M, Wilson C, Hose S, Bhutto IA, Grebe R, Dong A, Greenbaum S, Gu L, Sengupta S, Cano M, et al: Lysosomal-mediated waste clearance in retinal pigment epithelial cells is regulated by CRYBA1/ßA3/A1-crystallin via V-ATPase-MTORC1 signaling. Autophagy 10: 480-496, 2014.

26. Zhang CS, Jiang B, Li M, Zhu M, Peng Y, Zhang YL, Wu YQ, Li TY, Liang Y, Lu Z, et al: The lysosomal v-ATPase-ragulator complex is a common activator for AMPK and mTORC1, acting as a switch between catabolism and anabolism. Cell Metab 20: 526-540, 2014.

27. Cao X, Yang Q, Qin J, Zhao S, Li X, Fan J, Chen W, Zhou Y, Mao H and Yu X: V-ATPase promotes transforming growth factor- $\beta$-induced epithelial-mesenchymal transition of rat proximal tubular epithelial cells. Am J Physiol Renal Physiol 302: F1121-F1132, 2012.

28. Jung JY and Robinson CM: Interleukin-27 inhibits phagosomal acidification by blocking vacuolar ATPases. Cytokine 62: 202-205, 2013. 